\title{
Managed Care, Hospital Characteristics, and Inpatient Utilization for Sickle Cell Disease Patients
}

\author{
Eugene Lewit, $P h D^{1,2,3}$, Kavita Thirumalai, $M P H^{7}$, and C. Jason Wang, $M D, P h D^{1,2,3}$ \\ ${ }^{1}$ Center for Policy, Outcomes and Prevention and Division of General Pediatrics, Stanford University School of Medicine, Stanford, CA, USA; \\ ${ }^{2}$ Department of Health Research and Policy, Stanford University, Stanford, CA, USA; ${ }^{3}$ Center for Primary Care Outcomes Research, Department of \\ Medicine , Stanford University School of Medicine, Stanford, CA, USA.
}

KEY WORDS: managed care; sickle cell disease.

J Gen Intern Med 33(12):2053-5

DOI: $10.1007 / \mathrm{s} 11606-018-4630-4$

(c) Society of General Internal Medicine 2018

\section{INTRODUCTION}

Sickle cell disease (SCD) is associated with increased levels of hospitalization, morbidity, and mortality. ${ }^{1}$ Managed care is a strategy intended to reduce healthcare costs ${ }^{2}$ but no research exists on the effects of managed care on SCD patients. In this study, we examine the impact of managed care and hospital characteristics on utilization of inpatient care for SCD.

\section{METHODS}

We conducted a cross-sectional study using data from the California Office of Statewide Health Planning and Development (OSHPD) inpatient dataset for all patients admitted with primary and secondary diagnoses (ICD codes 282.60, 282.69, and 282.41, 282) of sickle cell disease in California in $2013 .^{3}$ A patient-level database was created for analysis using patient-specific record linking numbers to aggregate admissions data for each patient. Outcome measures include annual number of admissions (ADM) and mean-adjusted length of stay (LOS) per patient. Independent variables include gender, race, ethnicity, age, disease severity, patient socio-economic status, expected source of payment, hospital characteristics, and enrollment in managed care. Multivariate analyses using Stata 13 included Poisson regression for analysis of admissions (ADM) per patient per year and ordinary least squares for analysis of mean length of stay (LOS) per patient with each observation weighted by the number of admissions for the patient. Standard errors were adjusted for the clustering of patients in hospitals. Analyses were conducted for the entire sample and for the three age groups: $<21,21-32$, and $>32$-year-olds.

\section{RESULTS}

The 2722 patients in our sample had 7283 SCD-related admissions in 2013, a mean of 2.68 admissions per patient, and mean LOS per patient of 5.26 days (Table 1). Overall, $56 \%$ of patients were female, $83 \%$ were African-American, and $51 \%$ were of moderate income. Approximately $37 \%$ had severe disease and Medicaid was the primary source of coverage. Approximately $41 \%$ were treated in designated SCD centers and $50 \%$ were enrolled in managed care.

Adjusted for the other independent variables, we found no evidence that enrollment in managed care (IRR 1.09, 95\% CI 0.98-1.20) had any effect on the number of hospital admissions per patient in our sample nor on LOS (coef. 0.02, 95\% CI - 0.55-0.60) (Table 2).

There is some evidence that admission to a hospital that treats a high volume of SCD patients is associated with an increased number of visits per patient (IRR 1.14, 95\% CI 1.03-1.26) over the entire sample with an increase of approximately 1 day in mean LOS (coef. $0.88,95 \%$ CI $0.24-1.52$ ) and an even greater increase among those $>32$ years old (coef. $1.62,95 \%$ CI $0.66-2.57$ ).

For patients $<21$, admission to an SCD center is associated with increased LOS of 1.41 days (coef. 1.41, CI 0.28-2.54).

Published online August 20, 2018 
Table 1 Patient and Provider Characteristics and Hospital Utilization Data for SCD Patients in California in 2013

\begin{tabular}{|c|c|c|c|c|}
\hline & $\begin{array}{l}\text { All ages } \\
N(\%)\end{array}$ & $\begin{array}{l}<21 \text { years } \\
N(\%)\end{array}$ & $\begin{array}{l}21-32 \text { years } \\
N(\%)\end{array}$ & $\begin{array}{l}>32 \text { years } \\
N(\%)\end{array}$ \\
\hline Number of patients & 2722 & $865(31.8)$ & $782(28.7)$ & $1075(39.5)$ \\
\hline \multicolumn{5}{|l|}{ Patient characteristics } \\
\hline Female & $1522(55.9)$ & $424(49.0)$ & $435(55.6)$ & $663(61.7)$ \\
\hline \multicolumn{5}{|l|}{ Race/ethnicity } \\
\hline Hispanic & $236(8.7)$ & $103(11.9)$ & $61(7.8)$ & $72(6.7)$ \\
\hline Black & $2252(82.7)$ & $689(79.7)$ & $674(86.2)$ & $889(82.7)$ \\
\hline Other* & $234(8.6)$ & $73(8.4)$ & $47(6.0)$ & $114(10.6)$ \\
\hline \multicolumn{5}{|l|}{ Socio-economic status ${ }^{\dagger}$} \\
\hline Low-income & $1007(37.0)$ & $315(36.4)$ & $279(35.7)$ & $413(38.4)$ \\
\hline Moderate-income & $1388(51.0)$ & $459(53.1)$ & $400(51.2)$ & $529(49.2)$ \\
\hline High-income & $196(7.2)$ & $59(6.8)$ & $57(7.3)$ & $80(7.4)$ \\
\hline Severe disease ${ }^{\ddagger}$ & $1002(36.8)$ & $256(29.6)$ & $275(35.2)$ & $471(43.8)$ \\
\hline \multicolumn{5}{|l|}{ Provider characteristics } \\
\hline \multicolumn{5}{|l|}{ Hospital volume of SCD patients/year ${ }^{\S}$} \\
\hline Low & $888(32.6)$ & $121(14.0)$ & $307(39.3)$ & $460(42.8)$ \\
\hline Medium & $875(32.2)$ & $171(19.8)$ & $283(36.2)$ & $421(39.2)$ \\
\hline High & $959(35.2)$ & $573(66.2)$ & $192(24.6)$ & $194(18.0)$ \\
\hline SCD specialty center & $1112(40.9)$ & $656(75.8)$ & $204(26.1)$ & $252(23.4)$ \\
\hline \multirow[t]{2}{*}{ Managed care } & $1355(49.8)$ & $342(39.5)$ & $458(58.6)$ & $555(51.6)$ \\
\hline & All ages & $<21$ years & $21-32$ years & $>32$ years \\
\hline Payer categories & Mean (SD) & Mean (SD) & Mean (SD) & Mean (SD) \\
\hline Medicare & $0.172(0.377)$ & $0.006(0.076)$ & $0.154(0.362)$ & $0.319(.466)$ \\
\hline Med-Cal & $0.412(0.492)$ & $0.520(0.500)$ & $0.445(0.497)$ & $0.298(.468)$ \\
\hline Other gov. & $0.098(0.297)$ & $0.182(0.386)$ & $0.065(0.247)$ & $0.055(.215)$ \\
\hline Other payment & $0.004(0.061)$ & $0.002(0.048)$ & $0.006(0.080)$ & $0.003(.502)$ \\
\hline Self-pay & $0.042(0.200)$ & $0.024(0.154)$ & $0.058(0.233)$ & $0.044(.204)$ \\
\hline Private & $0.273(0.446)$ & $0.266(0.442)$ & $0.271(0.445)$ & $0.280(.449)$ \\
\hline \multirow[t]{2}{*}{ Outcomes } & Mean (SD) & Mean (SD) & Mean (SD) & Mean (SD) \\
\hline & Range & Range & Range & Range \\
\hline \multirow[t]{2}{*}{ Mean LOS (days) per patient } & $5.26(7.76)$ & $5.26(11.27)$ & $5.14(4.47)$ & $5.36(5.99)$ \\
\hline & $1-189$ & $1-189$ & $1-38$ & $1-69$ \\
\hline \multirow[t]{2}{*}{ Mean number of admissions per patient per year } & $2.68(3.12)$ & $2.27(2.18)$ & $3.34(4.02)$ & $2.52(2.96)$ \\
\hline & $1-34$ & $1-20$ & $1-34$ & $1-26$ \\
\hline
\end{tabular}

Italics represent major variable categories. For example, Recelethnicity is a category in the table that includes Hispanic, Black, Other "Includes White, Asian/Pacific Islander, Native America/Eskimo/Aleut, Other

${ }^{\dagger}$ Low-income (<200\% federal poverty level (FPL)); moderate-income (200\%-400\% FPL); high-income (> 400\% FPL)

${ }^{*}$ Additional diagnoses of cerebrovascular disease, acute chest syndrome, or renal failure (chronic or acute) present on at least one admission

${ }^{\S}$ Low-volume hospital ( $\leq 13$ SCD patients/year); moderate-volume hospital (13-40 SCD patients/year); high-volume hospital $(\geq 41$ SCD patients/year)

\section{DISCUSSION}

Our finding that managed care is not associated with reductions in hospital utilization for SCD patients in California may be noteworthy, given that others have reported improved access and quality of care for people with disabilities in Medicaid managed care. ${ }^{2}$ Our findings that admission to high SCD volume hospitals and specialty care centers is associated with increased hospital utilization are different from reports of improved outcomes associated with those hospital characteristics for other conditions. ${ }^{4}$ Specific to SCD, two previous studies reported inconsistent findings regarding hospital volume and LOS: one an inverse relationship between SCD-specific hospital volume ${ }^{5}$ and LOS and the other, the opposite. ${ }^{6}$ A caveat about our findings is that collinearity between hospital volume and SCD center variables made it difficult to measure the effects of each variable separately.

Considered as a whole, our findings suggest that it is time to consider new care coordination and other innovative strategies to reduce the burden of inpatient care for SCD patients.

Corresponding Author: C. Jason Wang, MD, PhD; Center for Primary Care Outcomes Research, Department of Medicine Stanford University School of Medicine, Stanford, CA, USA (e-mail: cjwang1@stanford.edu).

\section{Compliance with Ethical Standards:}

Conflict of Interest: The authors declare that they do not have a conflict of interest. 
Table 2 Multivariate Analyses of Factors Associated with Number of Hospital Admission Per Patient and Mean Length of Stay for Patients with Sickle Cell Disease Diagnosis, California 2013

\begin{tabular}{|c|c|c|c|c|c|c|c|c|}
\hline & \multicolumn{2}{|c|}{ Overall $(N=2722)$} & \multicolumn{2}{|c|}{$\begin{array}{l}\text { Age group: }<21 \text { years } \\
(N=865)\end{array}$} & \multicolumn{2}{|c|}{$\begin{array}{l}\text { Age group: } 21-32 \text { years } \\
(N=782)\end{array}$} & \multicolumn{2}{|c|}{$\begin{array}{l}\text { Age group: }>32 \text { years } \\
(N=1075)\end{array}$} \\
\hline \multicolumn{9}{|c|}{ Section 1: number of admissions per patient per year (estimated by Poisson regression) } \\
\hline Key independent variables & IRR & $95 \% \mathrm{CI}$ & IRR & $95 \% \mathrm{CI}$ & IRR & $95 \% \mathrm{CI}$ & IRR & $95 \% \mathrm{CI}$ \\
\hline Managed care & 1.09 & $0.98,1.20$ & 1.16 & $0.94,1.43$ & 1.17 & $0.94,1.46$ & 0.85 & $0.71,1.02$ \\
\hline High-volume hospital* & $1.14^{\dagger}$ & $1.03,1.26$ & NI & NI & 1.13 & $0.98,1.30$ & 1.05 & $0.88,1.26$ \\
\hline SCD center & NI & NI & 1.13 & $0.93,1.37$ & NI & NI & NI & NI \\
\hline \multicolumn{9}{|c|}{ Section 2: length of stay (LOS) per patient (estimated by weighted least squares regression) } \\
\hline Key independent variables & Coeff & $95 \% \mathrm{CI}$ & Coeff & $95 \% \mathrm{CI}$ & Coeff & $95 \% \mathrm{CI}$ & Coeff & $95 \% \mathrm{CI}$ \\
\hline Managed care & 0.02 & $-0.55,0.60$ & -0.44 & $-1.5,0.63$ & 0.22 & $-0.84,1.28$ & 0.009 & $-0.98,1.00$ \\
\hline High-volume hospital* & $0.88^{\dagger}$ & $0.24,1.52$ & NI & NI & 1.17 & $-0.007,2.35$ & $1.62^{\dagger}$ & $0.66,2.57$ \\
\hline SCD center & NI & NI & $1.41^{\dagger}$ & $0.28,2.54$ & NI & NI & NI & NI \\
\hline
\end{tabular}

Section 1: number of admissions per patient per year (estimated by Poisson regression)

Independent variables included in the overall model: severe disease ${ }^{\ddagger}$, Medicare ${ }^{\ddagger}$, Med-Cal ${ }^{\ddagger}$, other government ${ }^{\dagger}$, other payment, self-pay, managed care, high-volume hospital ${ }^{\dagger}$, male, Black ${ }^{\star}$, Hispanic, low-income ${ }^{\star}$, moderate-income, age group 21-32 $2^{\star}$, age group 33-64, and age group $\geq 65^{\star}$

Independent variables in the age group $<21$ model: severe disease ${ }^{\ddagger}$, Medicare, Med-Cal, other government ${ }^{\ddagger}$, other payment, self-pay, managed care, SCD center, male, Black ${ }^{\ddagger}$ Hispanic, low-income, moderate-income

Independent variables included in the age group 21-32 model: severe disease ${ }^{\ddagger}$, Medicare, Med-Cal ${ }^{\dagger}$, other government, other payment, self-pay ${ }^{\dagger}$, managed care, high-volume hospital, male, Black ${ }^{\dagger}$, Hispanic, low-income, moderate-income

Independent variables included in the age group $>32$ model: severe disease ${ }^{\ddagger}$, Medicare, Med-Cal ${ }^{\ddagger}$, other government, other payment, self-pay ${ }^{\dagger}$, managed care, high-volume hospital, male, Black ${ }^{\ddagger}$, Hispanic, low-income, moderate-income, age group $\geq 65^{\ddagger}$

Section 2: length of stay (LOS) per patient (estimated by weighted least squares regression)

Independent variables included in the overall model: severe disease ${ }^{\ddagger}$, Medicare ${ }^{\dagger}$, Med-Cal ${ }^{\dagger}$, other government, other payment, self-pay ${ }^{\dagger}$, managed care, high-volume hospital ${ }^{\dagger}$, male, Black, Hispanic, low-income, moderate-income, age group 21-32, age group 33-64, and age group $\geq 65$

Independent variables in the age group $<21$ model: severe disease ${ }^{\dagger}$, Medicare, Med-Cal, other government, other payment, self-pay, managed care, SCD center $^{\dagger}$, male, Black ${ }^{\ddagger}$, Hispanic, low-income, moderate-income,

Independent variables included in the age group 21-32 model: severe disease ${ }^{\dagger}$, Medicare ${ }^{\dagger}$, Med-Cal, other government, other payment, self-pay, managed care, high-volume hospital, male, Black, Hispanic, low-income, moderate-income

Independent variables included in the age group $>32$ model: severe disease ${ }^{\ddagger}$, Medicare ${ }^{\dagger}$, Med-Cal, other government, other payment, self-pay, managed care, high-volume hospital ${ }^{\dagger}$, male, Black, Hispanic, low-income, moderate-income, age group $\geq 65$

Severe disease indicates additional diagnoses of cerebrovascular disease, acute chest syndrome, or renal failure (chronic or acute) present on at least one admission. Low-income (<200\% federal poverty level (FPL)); medium-income (200-400\% FPL). Italics represent major variable categories. For example, Recelethnicity is a category in the table that includes Hispanic, Black, Other

IRR, incidence rate ratio; Coeff, coefficient

*High-volume hospital $(\geq 41$ patients per year)

${ }^{\dagger} P$ value $<0.05$

${ }^{*} P$ value $\leq 0.001$

\section{REFERENCES}

1. Brousseau DC, Owens PL, Mosso AL, Panepinto JA, Steiner CA. Acute care utilization and rehospitalizations for sickle cell disease. JAMA. 2010; 303 (13): 1288-1294. https://doi.org/10.1001/jama.2010.378

2. Highsmith N, Somers S. Adults with Disabilities in Medi-Cal Managed Care: Lessons from Other States, Medi-Cal Policy Institute, September 2003.

3. Office of Statewide Health Planning and Development. Patient Discharge Data (PDD) Data Dictionary.; 2015.
4. Morche J, Mathes T, Pieper D. Relationship between surgeon volume and outcomes: a systematic review of systematic reviews. Syst Rev. 2016. https://doi.org/10.1186/s13643-016-0376-4.

5. Mccavit TL, Lin H, Zhang S, Ahn C, Quinn CT, Flores G. Hospital volume, hospital teaching status, patient socioeconomic status, and outcomes in patients hospitalized with sickle cell disease. Am J Hematol. 2011. https:// doi.org/10.1002/ajh.21997.

6. Raphael JL, Richardson T, Hall M, et al. Association between hospital volume and within-hospital intensive care unit transfer for sickle cell disease in children's hospitals. J Pediatr. 2015. https://doi.org/10.1016/j. jpeds.2015.09.007. 